\title{
WAOA 2015 Special Issue on TOCS
}

\author{
Laura Sanità ${ }^{1}$ Martin Skutella ${ }^{2}$
}

Published online: 8 February 2018

(C) Springer Science+Business Media, LLC, part of Springer Nature 2018

This special issue of Theory of Computing Systems is devoted to selected papers from the 13th Workshop on Approximation and Online Algorithms (WAOA 2015), that took place in Patras, Greece, in September 2015. The guest editors selected 9 contributions presented at the conference, addressing fundamental topics related to approximation and online algorithms, which have been revised and expanded in this special issue.

In their article Constant-Time Local Computation Algorithms, Yishay Mansour, Boaz Patt-Shamir, and Shai Vardi give Local Computation Algorithms for several combinatorial optimization problems, whose time complexity is independent of the input size.

Sàndor P. Fekete, Kan Huang, Joseph S. B. Mitchell, Ojas Parekh, and Cynthia A. Phillips, in their contribution Geometric Hitting Set for Segments of Few Orientations, give new hardness and approximation results for several special cases of geometric hitting set problems, where the input consists of sets of line segments having a small number of distinct slopes.

In their paper Scheduling Parallel Jobs Online with Convex and Concave Parallelizability, Roozbeh Ebrahimi, Samuel McCauley, and Benjamin Moseley, consider the problem of scheduling jobs with convex or concave speedup curves in an online setting, and develop a new competitive algorithm for it.

Laura Sanità

1sanita@uwaterloo.ca

Martin Skutella

martin.skutella@tu-berlin.de

1 University of Waterloo, Waterloo, ON, Canada

2 Technische Universität Berlin, Berlin, Germany 
Katarzyna Paluch, in her contribution Maximum ATSP with Weights Zero and One via Half-Edges, gives a fast combinatorial 3/4-approximation algorithm for the maximum asymmetric TSP problem, with weights zero and one.

In their article Shortest Augmenting Paths for Online Matchings on Trees, Bartlomiej Bosek, Dariusz Leniowski, Piotr Sankowski, and Anna Zych-Pawlewicz, give a new bound on the total length of all augmenting paths found by the shortest augmenting path algorithm for online matchings on trees.

Nikhil Bansal, Marek Elias, Lukasz Jez, Grigorios Koumoutsos, and Kirk Pruhs, in their work Tight Bounds for Double Coverage Against Weak Adversaries, study the competitive ratio of the Double Coverage algorithm for the k-center problem in tree metrics, when compared against an offline algorithm which has less servers available.

On the Smoothness of Paging Algorithms, by Jan Reineke and Alejandro Salinger, is an article which introduces a notion of smoothness for paging algorithms, and derive bounds on the smoothness of some deterministic and randomized algorithms.

Shay Mozes and Eyal E. Skop, in their contribution Efficient Vertex-Label Distance Oracles for Planar Graphs, focus on computing data structures which can effectively answer distance queries in vertex-labeled planar graphs.

Approximation Algorithms for Connected Graph Factors of Minimum Weight, by Kamiel Cornelissen, Ruben Hoeksma, Bodo Manthey, N. S. Narayanaswamy, C. S. Rahul, and Marten Waanders, give new approximation algorithms for finding k-connected d-regular spanning subgraphs of minimum weight.

The guest editors would like to thank all the authors for their interesting contributions, and all the referees, whose remarkable work in the review process significantly improved the quality of this special issue. 